\title{
Molecular Docking of 4-Tert-buthyl-bis-(2,6-thiomorpholin-4-ylmethyl)-1-phenol (LQM319) on Fas Receptor (CD95)
}

\author{
Oscar Zuñiga ${ }^{1}$, Víctor H. Vázquez ${ }^{2}$, Ana María Velázquez ${ }^{2}$, Víctor H. Abrego ${ }^{2}$, \\ Sandra Díaz-Barriga Arceo", Patricia Ramírez ${ }^{1}$, Roberto Díaz ${ }^{1}$, Enrique Angeles ${ }^{2 *}$ \\ ${ }^{1}$ Laboratorio de Toxicología y Genética, Departamento de C. Biológicas, Facultad de Estudios Superiores Cuautitlán, Universidad \\ Nacional Autónoma de México, Cuautitlán Izcalli, México; ${ }^{2}$ Laboratorio de Química Medicinal Departamento de Ciencias Químicas \\ Facultad de Estudios Superiores Cuautitlán, Universidad Nacional Autónoma de México, Cuautitlán Izcalli, México. \\ Email: *angeles@unam.mx
}

Received November $14^{\text {th }}, 2012$; revised December $16^{\text {th }}, 2012$; accepted December $24^{\text {th }}, 2012$

\begin{abstract}
The balance between cell proliferation and cell growth characterizes tissue homeostasis on one side and cell death on the other side. Fas receptor-mediated apoptosis is a control mechanism for tissue homeostasis, and avoiding this death pathway predisposes to many human diseases, including cancer. Current therapies for this disease are invasive and do not have the desired effect in the control of the disease. In this context, the search for new drugs that contribute to a better treatment is gaining more relevance. 4-tert-butyl-bis-(2,6-thiomorpholin-4-ylmethyl)-1-phenol (LQM319) [1,2] is a drug currently in preclinical stage, and we have shown that it has a hypertensive effect, similar to captopril, in a hypertensive rat model. Different studies have shown that some chemicals that are used as antihypertensive agents have an antineoplastic effect against certain types of cancer, as is the case of hydralazine [3], and captopril [4], among others [5]. On the other hand, it has been reported that morpholine derivatives may activate Fas (CD95)-mediated apoptosis. The aim of the present study was to show the interaction between CD95 (receptor) and thiomorpholine derivatives (ligand) using molecular modeling and docking studies, and to elucidate the possible action mechanism of 4-tert-butyl-bis(2,6-thiomorpholin-4-ylmethyl)-1-phenol.
\end{abstract}

Keywords: Fas Receptor (CD95); Fas Ligand (FasL); Docking

\section{Introduction}

In response to stressful stimuli, cells usually mount a cellular stress response to ensure cell survival [6]. Under physiological conditions, this stress response limits tissue damage [7]. Apoptosis, or programmed cell death, is an evolutionarily conserved event that eliminates unwanted cells produced during animal development [8,9], and an important mechanism for eliminating tumorigenic cells. Apoptosis plays an important role in homeostasis, immune response, and elimination of abnormal cells [10]. Fas (CD95/Apo-1) has a central role in the physiological regulation of programmed cell death [11]. Apoptosis involves the activation of the pathways that lead to cell suicide by a characteristic sequence of events in which the cell becomes more compact, membrane blebbing occurs, chromatin becomes condensed, and DNA is fragmented [12]. Therefore, apoptosis evasion could be an important step possibly contributing to tumorigenesis.

*Corresponding author.
Apoptosis can be triggered by various stimuli from both outside and inside the cell; e.g., ligation of cell surface receptors, DNA damage, treatment with cytotoxic drugs or irradiation, and lack of survival signals [7]. A membrane receptor complex is formed in the apoptosis extrinsic pathway, followed by ligation of a member of the tumor necrosis factor receptor (TNFR) family [13]. Fas receptor and its ligand (FasL) are a pair of plasma membrane proteins whose interaction triggers one of the pathways for apoptosis. This intracellular cascade of events requires the Fas-associated death domain protein and the formation of death-inducing signaling complex, leading to caspase-8 activation and cell apoptosis $[14,15]$. The molecular pathway that regulates apoptosis will help in the investigation of novel cancer chemotherapeutic targets [16] offering, in turn, an opportunity to discover and develop new drugs [17].

Moreover, morpholine compounds based on phosphate derivatives, such as Amprenavir, are a new step towards the development of potent mimetic compounds of HIV-1 
protease inhibitors. It was also found that benzomorpholine is a PPAR $\gamma$ agonist, used in the treatment of Type II Diabetes (Rybczynski et al., 2004). On the other hand, evidence suggests that morpholine compounds have an antiproliferative effect [18-21]. LQM319 is a thiomorpholine compound that, as we showed in a previous report, has antihypertensive activity, similar to captopril [1]. Recent studies suggest that it also has an antiproliferative effect.

With this kind of evidence suggesting that the morpholine compound has biological activity, the aim of this study was to explore the potential mechanism by which LQM319 (Figure 1) could inhibit proliferation in transformed cells mediated by CD95.

\section{Material and Methods}

\subsection{Molecular Docking}

A structural model of the catalytic domain of CD95 was constructed using Sybyl, with the published magnetic resonance structure of $\mathrm{CD} 95$, and the modeling template was obtained from the Protein Data Bank. The active site was determinate using Sybyl and MOE software. In both cases, the protein active site was similar. LQM319 was modeled with Molecular Operating Environment (MOE) ver. 2010.10. A conformational analysis was performed using the Sybyl conformational analysis command, and the most stable ligand structure was docked into the CD95 binding pocket using the Sybyl DOCKING Protocol. The CD95 catalytic domain was downloaded from the protein data bank (www.pdb.org) with PDB-ID 3EZQ and $2.73 \AA$ resolution, and the pocket was determined by MOE's Site-Finder. The molecular structures studied by Masahiko et al. were modeled, minimized, and docked with the CD95 catalytic domain using MOE. For the conformational analysis, we used the stochastic method to avoid local minima for each molecule; whereas for the docking process, we used the protocol for rigidrigid docking, followed by rigid-flexible and flexibleflexible docking methods. Finally, the enzyme-ligand complex was visualized with MOE ver. 2010.10.

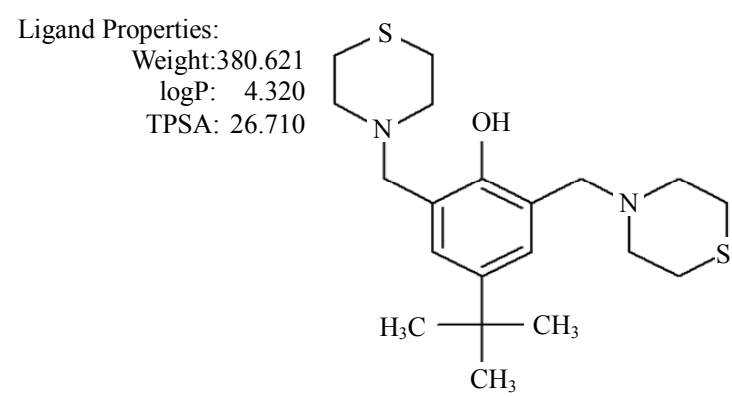

[4-tert-buthyl-bis-(2,6-thiomorpholin-4-ylmethyl)-1-phenol]

Figure 1. Chemical characteristics and structure of thiomorpholine compound.

\section{Results and Discussion}

Angiotensin-converting enzyme (ACE) is involved in hypertension, heart failure, myocardial infarction and diabetic nephropathy. Angiotensin is a vasoconstrictive peptide that directly influences the pathophysiology of coronary artery disease, playing a pivotal role in blood pressure regulation [22]. Captopril is a specific competetive inhibitor of angiotensin-converting enzyme, which is responsible for the conversion of angiotensin I to angiotensin II, and it has been suggested that it could inhibit cancer cells [23]. Captopril showed potent inhibition of Fas-induced apoptosis in a human lung epithelial cell line, and therefore it is also known as a possible lung fibrogenesis blocker [24]. This finding suggests that the conversion of ANG I to ANG II might be involved in Fas-induced apoptosis by ACE [23]. In this context, we used molecular docking between captopril and Fas receptor with MOE ver. 2010.10. The results showed a possible arrangement between captopril (ligand) and CD95 (receptor).

Based on previous reports suggesting that a group of morpholine compounds can modulate the synthesis of CD95 and that this regulation could trigger apoptosis $[19,21]$, and on a docking study conducted by Masahiko, et al., showing a favorable interaction between morpholine compounds and the receptor CD95 (Table 1 and Figures 2(a) and (b)), our hypothesis is that the LQM319 compound can bind to the CD95 receptor (also known as Fas receptor), and that this interaction could activate apoptosis in cancer cells. To check this hypothesis, docking studies were performed using SYBYL ver. 7.0. and MOE ver. 2010.10. The results showed a favorable interaction between the ligand (LQM319) and the CD95 active site. The amino acids that are more relevant to the molecular recognition process are: Lys 215, Tyr 216, Asp 301, Ser 304, Asn 308 (Figures 2(a) and (b)).

According to our results, the LQM319 compound has a great affinity (pKI) for Fas receptor (Table 2); the calculated efficiency is favorable, and the Gibbs free energy is negative. Also, based on this data, we can propose that interaction between the CD95 receptor and the LQM319 compound is possible and could induce programmed cell death or apoptosis.

On the other hand, we studied whether Fas receptor

Table 1. Table comparing the affinity and energy of the compounds studied.

\begin{tabular}{ccc}
\hline Compound & energy & affinity \\
\hline LY294002 & -11.982 & 5.258 \\
4-morpholino-2-phenylquinazolin-6-ol & -10.992 & 5.838
\end{tabular}

Energies from complexes with CD95 and morpholine compounds reported by Masahiko, computed with MOE. 


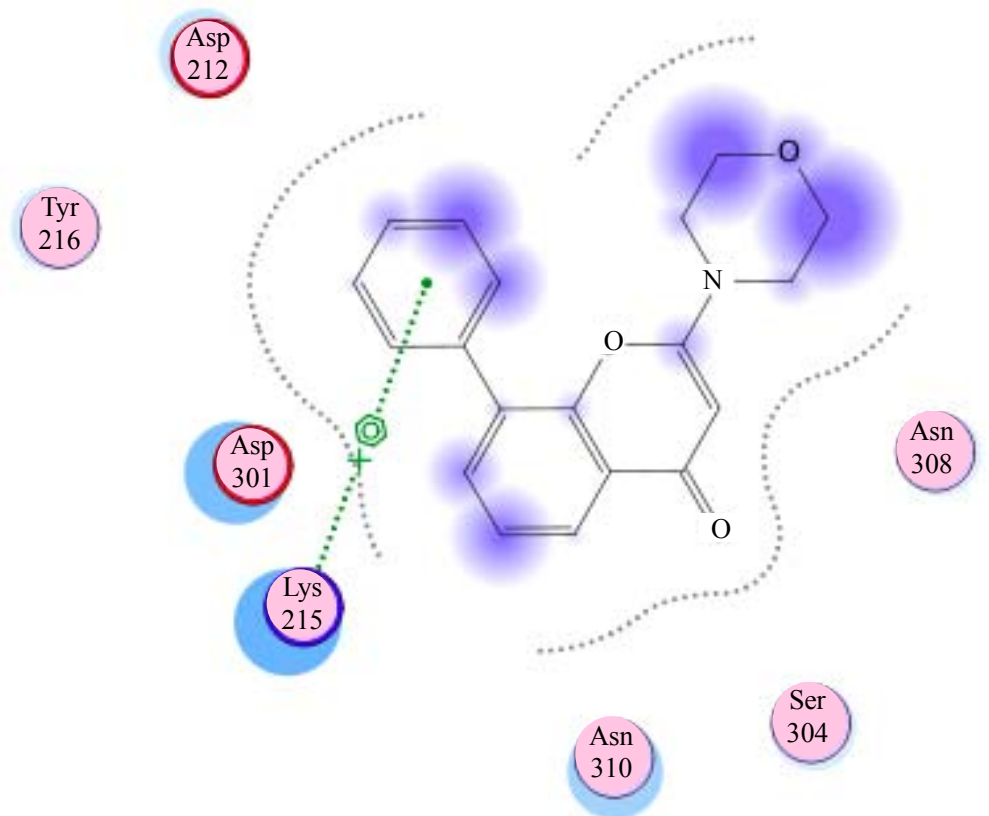

(a)

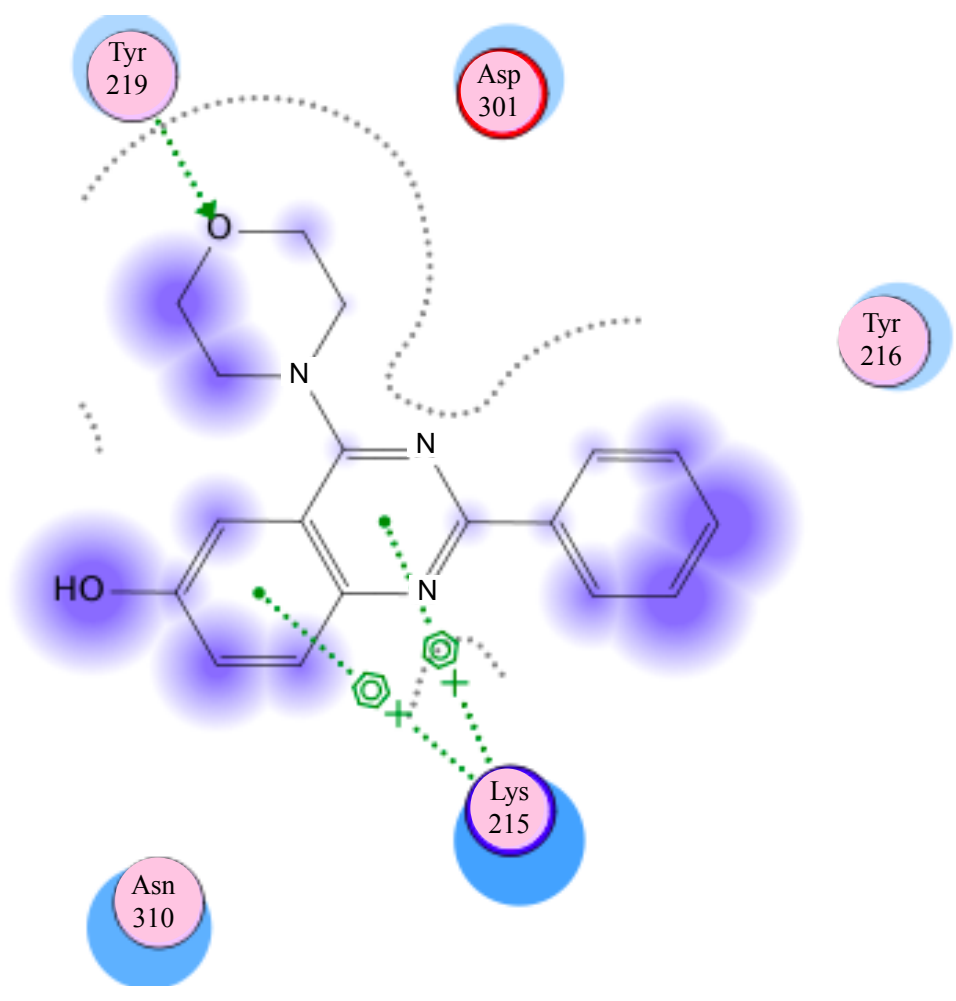

(b)

Figure 2. (a) Interaction of LY294002 with CD95; (b) Interaction of 4-morpholino-2-phenylquinazolin-6-ol with CD95.

Table 2. Table showing the energy and affinity of LQM319 compound.

\begin{tabular}{ccc}
\hline Compound & energy & affinity \\
\hline LQM319 & $5.5826 \pm 0.144$ & $-14.9332 \pm 1.574$ \\
\hline
\end{tabular}

Energy and affinity computed for the complex LQM319 and FAS receptor. could interact with captopril, an ACE inhibitor, since it has been reported by other studies to suppress rat hepatic fibrosis induced by pig serum [24] by blocking the renin angiotensin system (RAS) via angiotensin I converting enzyme (ACE) inhibition, thus reducing growth of colorectal cancer $(\mathrm{CRC})$ and liver metastases in a mouse 
model [25]. Captopril also induces Fas-mediated apoptosis of alveolar epithelial cells [23], but the results indicate that there are some interactions between the amino acids of the CD95 receptor pocket and captopril. The Gibbs free energy reaches a value of $-10.08 \mathrm{kcal} / \mathrm{mol}$, which indicates lower affinity between the two molecules (Figures 3 and 4).

\section{Conclusion}

The molecular docking results suggest a favorable interaction between the CD95 receptor (Apo-1 or Fas) and LQM319 (ligand) in the pocket of Fas receptor. Also, interactions between the CD95 receptor and the morpholine compounds reported by Masahiko were favorable, just as suggested by experimental studies. This helped us demonstrate that docking studies conducted

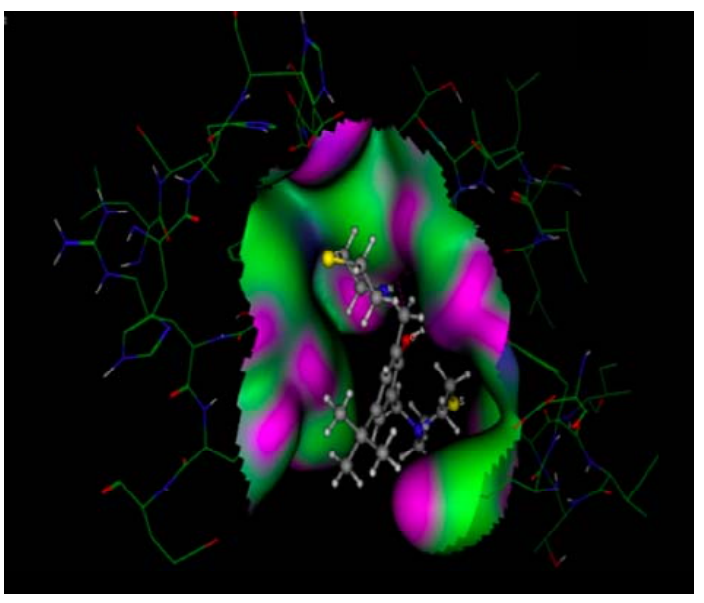

(a)
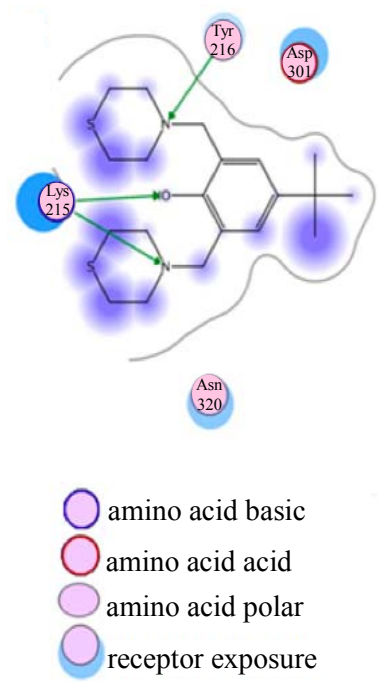

with LQM319 compound using the MOE program are reliable. We initially modeled LQM319's most stable conformation and reconstructed the CD95 pocket. The results indicate that the compound had a higher affinity for this protein (Table 1) than captopril and the morpholine compounds (LY294002, 4-morpholino-2-phenylquinazolin-6-ol). All the interactions that the complex showed prior to the docking study helped us to stabilize the entire system, as shown by the energy in table 2 . This suggests a more favorable complex between CD95 and LQM319 than that reported for captopril and the Masaiko morpholine compounds, and it might explain the antiproliferative effect of LQM319.

\section{Acknowledgements}

The authors would like to acknowledge PAPIIT/UNAM

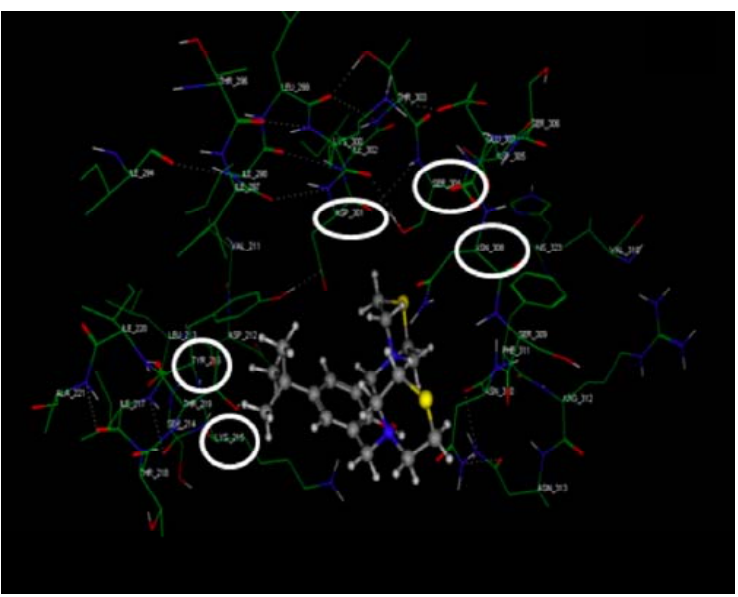

(b)
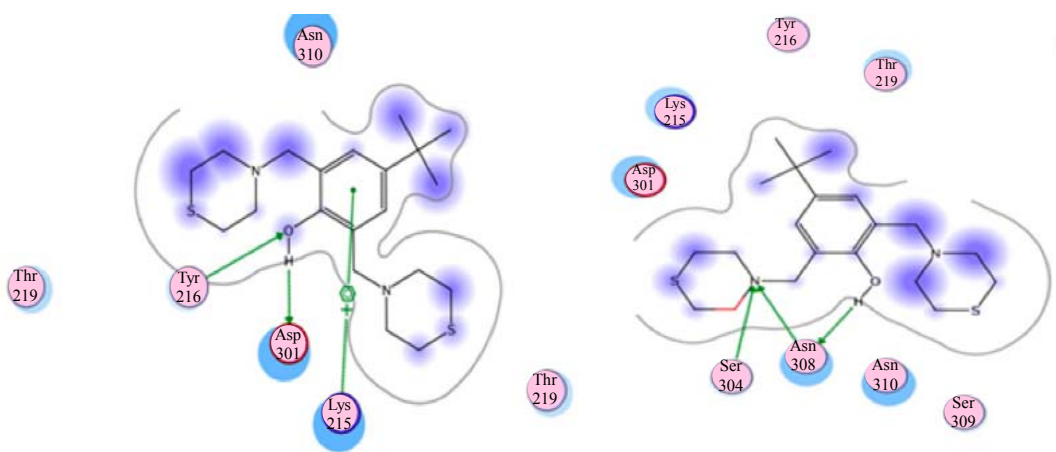

Ligand exposure

-... sidechain donor

- -..> sidechain receptor

Figure 3. Molecular Docking. Interactions between amino acids of active site of receptor CD95 and LQM319. The image (a) corresponding to formation of surface trought Gaussian contact of the CD95 receptor and LQM319 (ligand) the color green corresponding to hydrophobic region, the color violet corresponding to $\mathbf{H}$-Bonding and the blue color corresponding to mild Polar. The images (b) notes the Amino acids of CD95 receptor that interaction with the LQM319. (c) Representation of three docking between receptor CD95 and LQM319. 


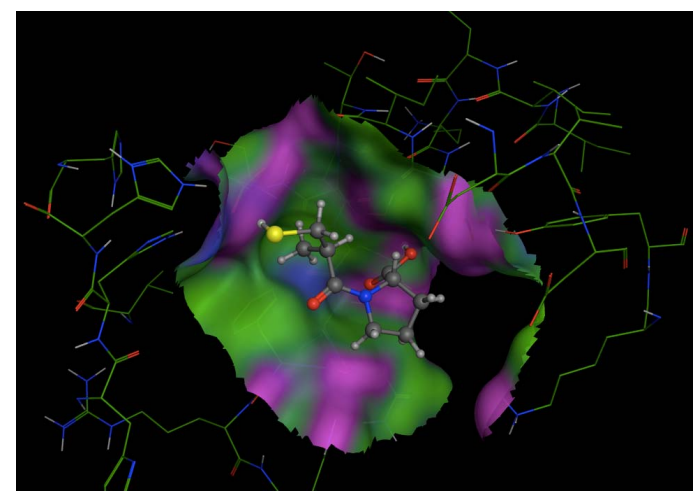

(a)

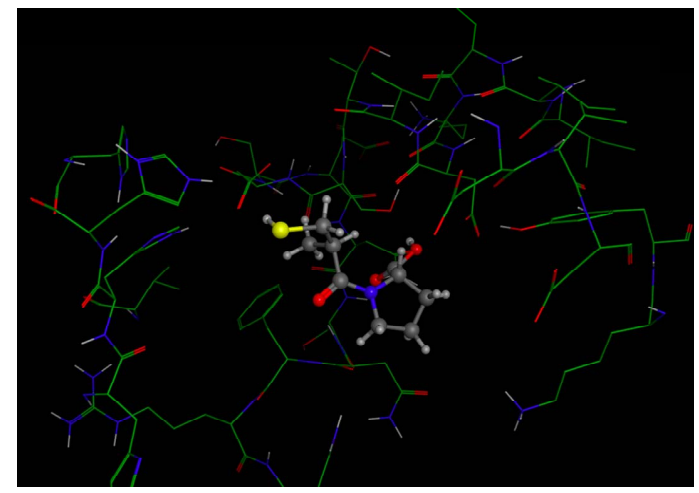

(b)
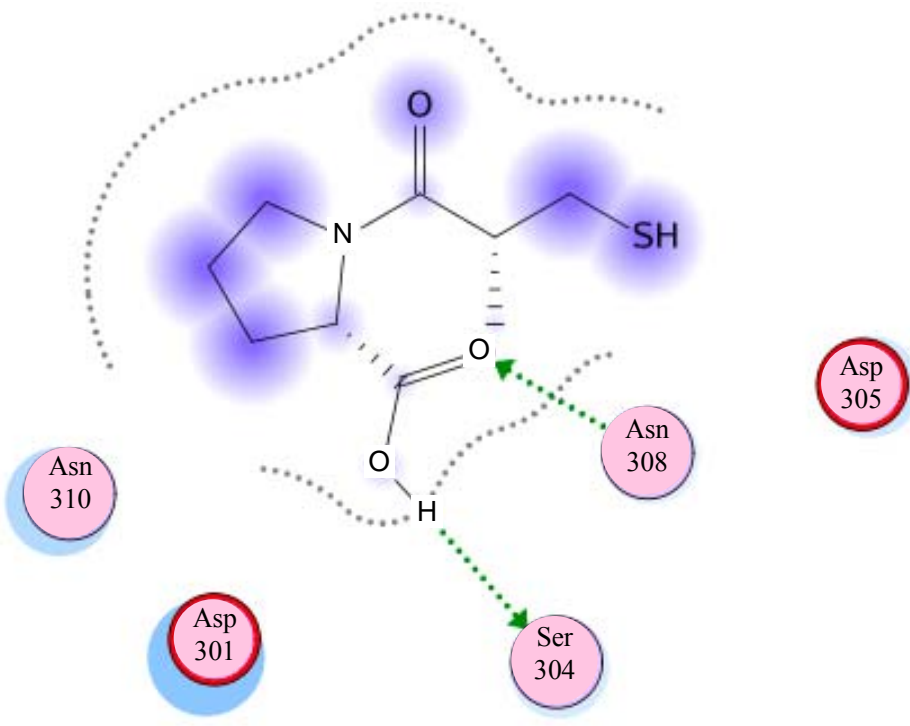

(c)

Figure 4. Molecular Docking. Between amino acids of active site of receptor CD95 and captopril. The image (a) corresponding to formation of surface trought Gaussian contact of the CD95 receptor and captopril (ligand) the color green corresponding to hydrophobic region, the color violet corresponding to $\mathrm{H}$-Bonding and the blue color corresponding to mild Polar. The images (b) notes the Amino acids of CD95 receptor that interaction with the captopril. (c) Representation of the docking between receptor $\mathrm{CD95}$ and captopril.

Projects No. IG200813 and IN207705, for partially supporting this work; F. Sotres, D. Jiménez, M. Hernandez, Rosa Ma. Valadez from FESC-UNAM, and Rosa I. del Villar M., Oscar Yañez and Georgina Duarte from USAI-UNAM, for their skillful technical assistance; DGTIC-UNAM for their support in using the Kan Balam super computer. This work was conducted as part of the Project Cátedra: Diseño de Sustancias Bioactivas of FESC-UNAM-2012.

\section{REFERENCES}

[1] A. Velázquez, L. Martínez, V. Abrego, M. A. Balboa, L. A. Torres, B. Camacho, S. Díaz-Barriga, A. Romero, R. López-Castañares and E. Angeles, "Synthesis and Antihypertensive Effects of New Methylthiomorpholinphenol Derivatives," European Journal of Medicinal Chemistry, Vol.43, No. 3, 2008, pp. 486-500.

\section{doi:10.1016/i.ejmech.2007.04.003}

[2] A. Ma. Velázquez, G. Díaz, A. Ramírez, R. Hernández, H. Santillán L. Martínez, V. Abrego, M.A. Balboa, L.A. Torres, B. Camacho, S. Díaz-Barriga, A. Romero, R. López-Castañares, A. Dueñas-González, G. Cabrera and E. Angeles, "A Novel One Pot, Solvent-Free Mannich Synthesis of Methylpiperidinyl Phenols, Methylphenylmorpholinyl Phenols and Methylthiophenylmorpholinyl Phenols Using Infrared Light Irradiation," Arkivoc, Vol. ii, No. 2006, pp. 150-161.

[3] E. Angeles, V. H. Vázquez, O. Vázquez, A. Ma. Velázquez, A. Ramírez, L. Martínez, S. Díaz-Barriga, A. Romero, G. Cabrera, R. López-Castañares and A. Dueñas, "Computational Studies of 1-Hydrazinophthalazine (Hydralazine) as an Antineoplasic Agent. Docking Studies on Methyltransferase," Letters in Drug Design and Discovery, Vol. 2, No. 4, 2005, pp. 282-286. doi: $10.2174 / 1570180054038413$

[4] K. Hudson and G. Ronquist, "Medical Use of Captopril 
for the Treatment and Prophylaxis of Cancer," Patent No.WO2003077909, 2003.

[5] Y. K. Chae, M. E. Valsecchi, J. Kim, A. L. Bianchi, D. Khemasuwan, A. Desai and W. Tester, "Therapeutic Combination and Methods of Treatment with a dll4 Antagonist and an Anti-Hypertensive Agent," Cancer Investigation, Vol. 29, No. 9, 2011, pp. 585-593. doi:10.3109/07357907.2011.616252

[6] D. Kültz, "Molecular and Evolutionary Basis of the Cellular Stress Response," Annual Review of Physiology, Vol. 67,2005 , pp. $225-257$. doi:10.1146/annurev.physiol.67.040403.103635

[7] S. Fulda and K. M. Debatin, "Extrinsic versus Intrinsic Apoptosis Pathways in Anticancer Chemotherapy," Oncogene, Vol. 25, No. 34, 2006, pp. 4798-4811. doi:10.1038/sj.onc. 1209608

[8] V. Zuzarte-Luis and J. M. Hurle, "Programmed Cell Death in the Developing Limb," The International Journal of Developmental Biology, Vol. 6, No. 7, 2002, pp. 871-876.

[9] P. Meier, A. Finch and G. Evan, "Apoptosis in Development," Nature, Vol. 407, No. 6805, 2000, pp. 796-801. doi: $10.1038 / 35037734$

[10] M. Raff, "Cell Suicide for Beginners," Nature, Vol. 396, No. 6707, 1998, pp.119-122. doi:10.1038/24055

[11] P. Krammer, "CD95's Deadly Mission in the Immune System," Nature, Vol. 407, No. 6805, 2000, pp. 789-795. doi: $10.1038 / 35037728$

[12] A. Saraste and K. Pulkki, "Morphologic and Biochemical Hallmarks of Apoptosis," Cardiovascular Research, Vol. 45, No. 3, 2000, pp. 528-37. doi:10.1016/S0008-6363(99)00384-3

[13] U. Sartorius, I. Schmitz and P. Krammer, "Molecular Mechanisms of Death-Receptor-Mediated Apoptosis," ChemBioChem, Vol. 2, No. 1, 2001, pp. 20-29. doi:10.1002/1439-7633(20010105)2:1<20::AID-CBIC20 $>3.0 . \mathrm{CO} ; 2-\mathrm{X}$

[14] M. Lenardo, K. Chan, F. Hornung, H. McFarland, R. Siegel, J. Wang and L. Zheng, "Mature T Lymphocyte Apoptosis: Immune Regulation in a Dynamic and Unpredictable Antigenic Environmental," Annual Review of Immunology, Vol. 17, 1999, pp. 221-253. doi:10.1146/annurev.immunol.17.1.221

[15] S. Nagata, "Fas Ligand-Induced Apoptosis," Annual Review of Genetics, Vol. 33, 1999, pp. 29-55. doi:10.1146/annurev.genet.33.1.29

[16] K. Bailey, H. Cook and C. McMaster, "The Phospholipid Scramblase PLSCR1 Increases UV Induced Apoptosis Primarily through the Augmentation of the Intrinsic Apoptotic Pathway and Independent of Direct Phosphorylation by Protein Kinase C $\delta$," Biochimica et Biophysica Acta, Vol. 1733, No. 2-3, 2005, pp. 199-209. doi:10.1016/i.bbalip.2004.12.013

[17] J. J. Alam, “Apoptosis: Target for Novel Drugs," Trends in Biotechnology, Vol. 21, No. 11, 2003, pp. 479-483. doi:10.1016/i.tibtech.2003.08.006

[18] J. A. Folkes, K. Ahmadi, K. Alderton, S. Alix, J. S. Baker, G. Box, S. Chuckowree, A. Clarke, P. Depledge, A. S.
Eccles, S. Friedman, A. Hayes, C. Hancox, A. Kugendradas, L. Lensun, P. Moore, G. A. Olivero, J. Pang, S. Patel, H. G. Pergl-Wilson, I. F. Raynaud, A. Robson, N. Saghir, L. Salphati, S. Sohal, M. H. Ultsch, M. Valenti, H. J. Wallweber, N. C. Wan and C. Wiesmann, "The Identification of 2-(1H-indazol-4-yl)-6-(4-methanesulfonyl-piperazin-1-ylmethyl)-4-morpholin-4-yl-thieno[3,2-d]pyrim idine (GDC-0941) as a Potent, Selective, Orally Bioavailable Inhibitor of Class I PI3 Kinase for the Treatment of Cancer," Journal of Medicinal Chemistry, Vol. 51, No. 18, 2008, pp. 5522-5532. doi:10.1021/jm800295d

[19] H. Qiuxia, Z. Xingshang, S. Mei, Z. Jing, Z. Shangli and M. Junying, "Novel Morpholin-3-One Derivatives Induced Apoptosis and Elevated the Level of P53 and Fas in A549 Lung Cancer Cells," Bioorganic \& Medicinal Chemistry, Vol. 15, No. 11, 2007, pp. 3889-3895. doi:10.1016/j.bmc.2007.03.008

[20] M. Hayakawa, H. Kaizawa, H. Moritomo, T. Koizumi, T. Ohishi, M. Okada, S. Tsukamoto, P. Parker, P. Workman and M. Waterfield, "Synthesis and Biological Evaluation of 4-Morpholino-2-phenylquinazolines and Related Derivatives as Novel PI3 Kinase p1 $10 \alpha$ Inhibitors," Bioorganic \& Medicinal Chemistry, Vol. 14, No. 20, 2006, pp. 6847-6858. doi:10.1016/j.bmc.2006.06.046

[21] D. Yin, M. Woodruff, Y. Zhang, S Whaley, J. Miao, K. Ferslew, J. Zhao and C. Stuart, "Morphine Promotes Jurkat Cell Apoptosis through Pro-Apoptotic FADD/P53 and Anti-Apoptotic PI3K/Akt/NF-nB Pathways," Journal of Neuroimmunology, Vol. 174, No. 1, 2006, pp. 101-107. doi:10.1016/i.jneuroim.2006.02.001

[22] V. Dzau, A. J. Folkes, K. Ahmadi, W. K. Alderton, S. Alix, S. J. Baker, G. Box, I. S. Chuckowree, P. A. Clarke, P. Depledge, S. A. Eccles, L. S. Friedman, A. Hayes, T. C. Hancox, A. Kugendradas, L. Lensun, P. Moore, A. G. Olivero, J. Pang, S. Patel, G. H. Pergl-Wilson, F. I. Raynaud, A. Robson, N. Saghir, L. Salphati, S. Sohal, M. H. Ultsch, M. Valenti, H. J. Wallweber, N. C. Wan, C. Wiesmann, P. Workman, A. Zhyvoloup, M. J. Zvelebil and S. J. Shuttleworth, "The Identification of 2-(1H-Indazol4-yl)-6-(4-Methanesulfonyh-piperazin-1-ylmethyl)-4-morpholin-4-yl-thieno[3,2-d]pyperidine (GDC-0941) as a Potent, Selective, Orally Bioavailable Inhibitor of Class I PI3 Kinase for the Treatment of Cancer," Journal of Medicinal Chemistry, Vol. 51, No. 18, 1994, pp. 5522-5532.

[23] R. Wang, A. Zagariya, E. Ang, O. Ibarra-Sunga and B. D. Uhal, "Fas-Induced Apoptosis of Alveolar Epithelial Cells Requires ANG. II II Generation and Receptor Interaction," American Journal of Physiology. Lung Cellular and Molecular Physiology, Vol. 277, Pt. 1, 1999, pp. 1245-1250.

[24] A. Molteni, W. Ward, C. Ts'ao, N. Solliday and M. Dunne, "Monocrotaline-Induced Pulmonary Fibrosis in Rats: Amelioration by Captopril and Penicillamine," Proceedings of the Society for Experimental Biology and Medicine, Vol. 180, No. 1, 1985, pp. 112-120.

[25] H. J. Neo, I. E. Ager, W. P. Angus, J. Zhu, B. C. Herath and C. Christophi, "Changes in the Renin Angiotensin System during the Development of Colorectal Cancer Liver Metastases," Cancer, Vol. 10, No. 134, 2010, pp. $1-11$. 